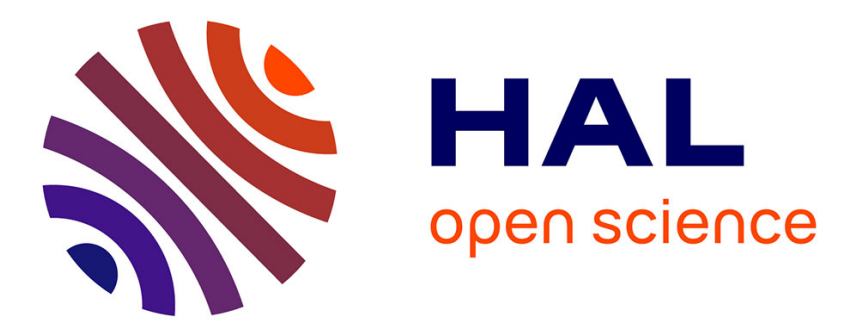

\title{
Distributed Source Seeking without Global Position Information
}

\author{
Ruggero Fabbiano, Federica Garin, Carlos Canudas de Wit
}

\section{To cite this version:}

Ruggero Fabbiano, Federica Garin, Carlos Canudas de Wit. Distributed Source Seeking without Global Position Information. IEEE Transactions on Control of Network Systems, 2018, 5 (1), pp.228238. 10.1109/TCNS.2016.2594493 . hal-01354294

\section{HAL Id: hal-01354294 https://hal.science/hal-01354294}

Submitted on 18 Aug 2016

HAL is a multi-disciplinary open access archive for the deposit and dissemination of scientific research documents, whether they are published or not. The documents may come from teaching and research institutions in France or abroad, or from public or private research centers.
L'archive ouverte pluridisciplinaire HAL, est destinée au dépôt et à la diffusion de documents scientifiques de niveau recherche, publiés ou non, émanant des établissements d'enseignement et de recherche français ou étrangers, des laboratoires publics ou privés. 


\title{
Distributed Source Seeking without Global Position Information
}

\author{
Ruggero Fabbiano, Federica Garin, Carlos Canudas de Wit
}

\begin{abstract}
We present a distributed control law to steer a group of autonomous communicating sensors towards the source of a diffusion process. The graph describing the communication links between sensors has a time-invariant topology, and each sensor is able to measure (in addition to the quantity of interest) only the relative bearing angle with respect to its neighbour, but has no absolute position information and does not know any relative distance. Using multiple sensors is useful in wide environments (e.g., under the sea), or when the function describing the diffusion process is slowly changing in space, so that a single sensor may have to travel long distances before having a good gradient estimation. Our approach is based on a twofold control law, which is able to bring and keep the set of sensors on a circular equispaced formation, and to steer the circular formation towards the source via a gradient-ascent technique. The effectiveness of the proposed algorithm is both theoretically proven and supported by simulation results.
\end{abstract}

Index Terms-Source seeking, Distributed control, Formation control, Gradient search, Poisson integral, Sensor networks.

\section{INTRODUCTION}

$\mathbf{T}$ HE source seeking problem consists in localizing a source emitting from an unknown position, by exploiting the fact that the concentration of the emitted quantity is higher near the source and decays with distance. This problem arises in various applications, e.g., detection of pollutants, explosives, drugs, or localization of sound, heat or vent sources.

In recent years, leveraging the richness of results on collaborative sensing, distributed control and multi-agents formations, a new line of research has emerged, focusing on multi-agent source seeking, where multiple mobile sensors collaborate in order to find the source, improving over single-sensor techniques, e.g., by allowing faster convergence, exploration of a wider area, robustness to errors and failures.

One of the first contributions is [1], where it is assumed that each vehicle can measure the full gradient, and the authors develop a twofold algorithm with a gradient-descent term and inter-vehicle forcing terms. Other early contributions are [2] and [3]. In the former, formation control uses potential functions to drive agents in a desired formation moving along a virtual body, whose gradient-climbing trajectory depends on a computation of the gradient obtained filtering all sensor measurements. In [3], the authors propose to solve the sourcelocalization problem with distributed optimization algorithms making use of measurements from sensors spread all over the

R. Fabbiano was and F. Garin is with INRIA (NeCS team, Inria Grenoble Rhône-Alpes) and GIPSA-lab, Grenoble, France; e-mails: ruggero.fabbianodsfr.fr, federica.garindinria.fr.

C. Canudas-de-Wit is with CNRS, GIPSA-lab (NeCS team), Grenoble, France; e-mail: carlos. canudas-de-witegipsa-lab.fr. region of interest, and not moving towards the source. Papers [4], [5] have introduced a collaborative control law to steer a fleet of AUVs (autonomous underwater vehicles) to the source of a signal distribution using only direct signal measurements, by arranging the AUVs in a circular formation and using measurements along the circle to get a gradient approximation. The formation control needed to achieve this goal has been further studied in [6]. The gradient approximation formula has been studied in [7], giving a mathematical framework for its validity and an extension to the 3-dimensional case; in this paper, though, gradient ascent was performed in a centralized way, with a single agent using measurements from all sensors. The recent paper [8] builds upon ideas from [5] to perform an improved collaborative search, with a Newton-Raphson method.

A gradient-based method is also considered in [9], where the authors propose two algorithms, based on the estimation of the gradient at the formation centroid, with different techniques for the two cases where the function describing the spatial distribution of the measured quantity is known or unknown; then, stochastic approximation is used to ensure convergence.

In [10], the gradient estimation makes use also of time series of measurements, instead of instantaneous measurements only, so that agents can also have an individual local gradient direction, and alternate phases of individual exploration with a collaborative exploration in formation.

Another class of algorithms are extremum seeking ones, where the gradient direction is indirectly estimated from the measurements, by probing the environment with suitable movements. In the early work [11], a leader agent performs extremum seeking, while all other agents are controlled to behave as followers. In [12], distributed formation control is combined with a centralized extremum seeking controller, which gives a gradient-ascent reference to the formation.

Most of these algorithms make explicit use of the absolute position of the sensors. A notable exception is [9], where the formation control and gradient estimation rather rely on relative localization, with respect to other sensors and to the formation centre.

\section{Paper Contribution}

We propose a distributed algorithm to estimate the gradient of a function describing a diffusion process, and use this information to drive a set of moving sensors towards the source. The effectiveness of such an algorithm is both theoretically proven and supported by simulation results.

The framework is close to the one of previous works [4], [5], [8], but our contribution differs as we avoid using full 
position information, which may be unavailable in various operating environments such as underwater or indoor vehicles, or in applications where inertial navigation systems are too expensive or not sufficiently accurate. We only suppose the sensors to be able to measure the relative angle with respect to their neighbours, differently from all above-mentioned distributed source seeking methods, which require either the knowledge of sensors absolute position, or at least relative localization. The main motivation for the restriction to relative angles is that in vision-based navigation angles are much easier to obtain than distances, see e.g. [13] for an example of bearing-angle sensor. We have already considered the same assumption in our preliminary work [14]; in that paper, though, our twofold objective (formation control and motion towards the source) was expressed in an antagonistic control law, which, together with a formation control algorithm without theoretical stability guarantees [15], resulted in an algorithm with slower convergence in simulation and no theoretical convergence proof. In this paper we introduce and study a new algorithm, where the formation control is based on techniques from [16], together with a suitable change of variables and a distributed gradient-approximation algorithm, so that we can express the global objective of driving the vehicles to a circular formation whose centre moves along the gradient direction, by making use only of the limited local information.

\section{Notation}

We will use the notation $z=\rho \mathrm{e}^{\mathrm{i} \theta}$ to denote the point $(x, y)=\rho(\cos \theta, \sin \theta) \in \mathbb{R}^{2}$, using the usual bijection $z=(x, y)$ between points in $\mathbb{R}^{2}$ and elements of $\mathbb{C}$. Multiplication $\mathrm{e}^{\mathrm{i} \alpha} z$ will denote rotation of vector $z$ by an angle $\alpha$ (counterclockwise for $\alpha>0$ ), as in usual multiplication of complex numbers. However, all other operations will be intended as operations on reals and on vectors in $\mathbb{R}^{2}$; in particular, all derivatives are intended as (partial) derivatives of real-valued functions, and integrals are intended as entry-wise integrals of the real-valued entries of the vectors. $\nabla f$ is the gradient of the function $f$, and $\nabla^{2}$ is the Laplacian operator. $\partial \Omega$ denotes the border of an open set $\Omega$, and its closure is denoted by $\bar{\Omega}=\Omega \cup \partial \Omega . B_{r}\left(z_{\mathrm{c}}\right)$ indicates the circle of radius $r$ centered in $z_{\mathrm{c}}$. Sums of sensor indexes $1, \ldots, n$ are always intended $\bmod n$, and sums of angles are $\bmod 2 \pi$.

\section{PROBlem Formulation}

\section{A. Autonomous Sensors}

We consider a group of $n$ communicating sensors moving in a region $\Omega \subset \mathbb{R}^{2}$, where a diffusion process is taking place, with the nonholonomic kinematics

$$
\begin{aligned}
\dot{z}_{i}(t) & =v_{i}(t) \mathrm{e}^{\mathrm{i} \theta_{i}(t)} \\
{\left[\begin{array}{c}
v_{i}(t) \\
\dot{\theta}_{i}(t)
\end{array}\right] } & =\boldsymbol{u}_{i}(t),
\end{aligned}
$$

where $z(t)$ is the rotational point of the sensor in the plane, and $\theta(t)$ is its heading angle. The sensors, which can take pointwise measurements of the emitted quantity, have no position information, but know the value of $n$ and are able to measure the bearing angle with respect to their following

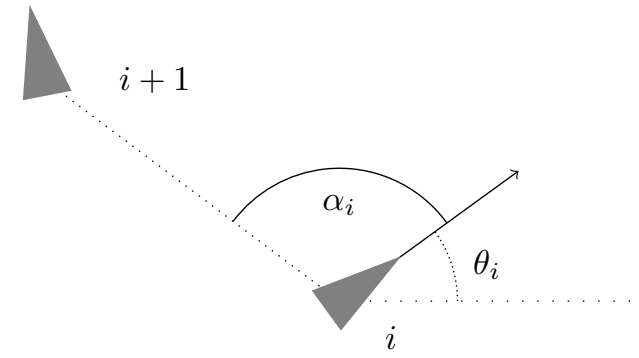

Fig. 1: Relative bearing angle $\alpha_{i}$
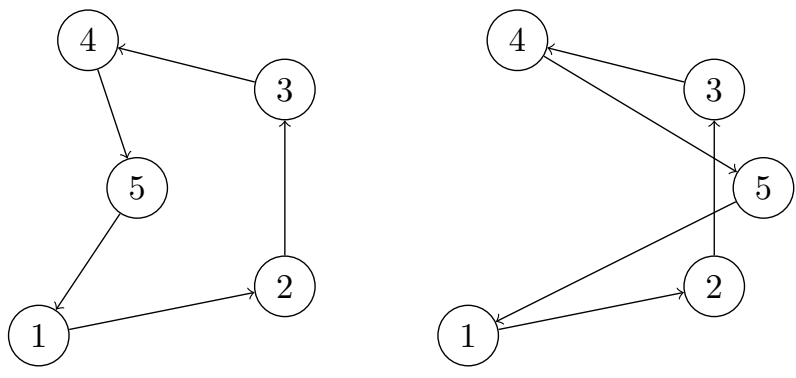

Fig. 2: Examples of ring communication network.

neighbour (i.e., the angle between the orientation of an agent and the vector from itself to its neighbour, as explained in Fig. 1), defined by

$$
\alpha_{i}(t)=\arg \left(z_{i+1}(t)-z_{i}(t)\right)-\theta_{i}(t) .
$$

We consider a ring communication network, where sensor $i$ can receive messages sent by sensor $i-1$, irrespective of the sensors position and of time (see Fig. 2). This assumption is not essential (other communication strategies are possible), but is natural when the sensors are not too far from a circular formation, as it will be the case in this paper. Indeed, we will show that a circular formation with sensors equispaced along the circle is desirable in order to compute a gradient approximation from their measurements, and we will describe a suitable control law which ensures that such a formation is obtained (from initial conditions not too far from this equilibrium) and maintained while the formation centroid is driven towards the source.

\section{B. Diffusion Process}

We consider steady-state behaviors of homogeneous diffusion processes caused by an isotropic source emitting at a constant rate. Such a process is governed by the well-known diffusion equation

$$
\frac{\partial f(z, t)}{\partial t}-c \nabla^{2} f(z, t)=0, \forall z \in \Omega, t \geq 0,
$$

where $f$ is the concentration variable, $c$ is a diffusion coefficient, and $\Omega \subset \mathbb{R}^{2}$. In particular, as depicted in Fig. 3, we consider the region of interest $\tilde{\Omega} \subset \mathbb{R}^{2}$ as a connected bounded set $\tilde{\Omega}=\Omega \cup \Omega_{\mathrm{s}}$, where $\Omega_{\mathrm{s}}$ is the connected bounded set that identifies the source, and therefore we have that $\partial \Omega=\partial \tilde{\Omega} \cup \partial \Omega_{\mathrm{s}}$. The values of $f$ on the inner boundary $\partial \Omega_{\mathrm{s}}$ are imposed by the source, so we can assume that values of $f$ on $\partial \Omega_{\mathrm{s}}$ are higher than the ones on $\partial \tilde{\Omega}$. As we will 


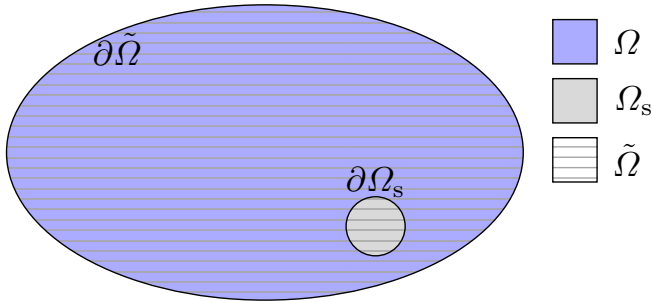

Fig. 3: Sets as defined in Section II: $\Omega=\tilde{\Omega} \backslash \Omega_{\mathrm{s}}$, and $\partial \Omega=$ $\partial \tilde{\Omega} \cup \partial \Omega_{\mathrm{s}}$.

see below, we have that $\max f(z) \in \partial \Omega$, that means, for our previous consideration, that it lies on $\partial \Omega_{\mathrm{s}}$; our source localisation problem is then mathematically equivalent to the problem of finding the maximum of $f$.

Once the steady-state has been reached, supposing that the source is still emitting at a constant rate (this happens in many cases of practical interest, e.g., in a heating process, or in the dispersion of a chemical substance), or that possible source variations are slow in the time-scale of interest, the diffusion equation (3) can be approximated by the Laplace equation, i.e.,

$$
\nabla^{2} f(z)=0, \forall z \in \Omega,
$$

whose solutions are called "harmonic functions" and have many mathematical properties which can be exploited to devise a gradient-ascent source seeking.

\section{Source-SEeking Strategy}

\section{A. Gradient ascent}

The source-localisation problem, formulated in previous section as the problem of finding the maximum of a harmonic function $f$, can be solved by a gradient ascent algorithm. We refer the reader to our previos work [7] for a detailed discussion, while we briefly recall here the main results, which will be used in the remainder of this paper as a tool for constructing a distributed source-seeking algorithm.

Harmonic functions satisfy the so-called local maximum principle, which ensures that (except for the trivial case of a constant function), $f$ can have maxima only on the border $\partial \Omega$, not in the interior of $\Omega$. This justifies the use of a gradient ascent technique, despite the fact that harmonic functions might not be convex.

Then, harmonicity of $f$ ensures the possibility to determine $f$ and all derivatives of $f$ at a point $z_{c}$, using measurements along a curve encircling $z_{c}$. In particular, in the case where the curve is a circle, explicit integral formulae exist, called Poisson integrals for the circle. We will make use of a Poisson integral to find the gradient $f\left(z_{c}\right)$, based on the values of $f$ along a circle centered in $z_{c}$, as follows:

$$
\nabla f\left(z_{\mathrm{c}}\right)=\frac{1}{\pi r} \int_{0}^{2 \pi} \mathrm{e}^{\mathrm{i} \theta} f\left(z_{\mathrm{c}}+r \mathrm{e}^{\mathrm{i} \theta}\right) \mathrm{d} \theta .
$$

In the following, we will approximate the above integral with a finite sum of measurements along the circle, taken by sensors, while a suitably designed control law will steer the sensors in a circular formation whose centre moves in the direction of the computed gradient.

As discussed in [5], [7], the Poisson formula (5), which holds exactly for harmonic functions, can also be used to approximate the gradient of more general functions, under the only requirement of $\mathcal{C}^{1}$ regularity. Hence, the distributed source-seeking method developed in this paper can be applied to some more general sources, provided they have enough regularity to justify the gradient approximation and the gradient ascent technique.

\section{B. Control Law}

We need to develop an algorithm which is able to both steer the sensors towards the gradient direction, and bring them on a circular formation, such that the gradient itself is correctly estimated.

We introduce a control law assuming that each sensor $i$ is able to compute the difference $\bar{\theta}_{i}(t)=\theta_{\text {ref }}(t)-\theta_{i}(t)$, where $\theta_{\text {ref }}$ is a common reference (we will discuss in Section IV how to obtain it). Let $v_{\mathrm{c}}>0$ and $v_{\mathrm{r}}>v_{\mathrm{c}}$, with $\bar{v}=\frac{v_{\mathrm{c}}}{v_{\mathrm{r}}}$, be fixed parameters; we propose the following control law:

$$
\begin{aligned}
& v_{i}(t)=v_{\mathrm{c}} \cos \bar{\theta}_{i}(t)+\sqrt{v_{\mathrm{r}}^{2}-v_{\mathrm{c}}^{2} \sin ^{2} \bar{\theta}_{i}(t)} \\
& \dot{\theta}_{i}(t)=b_{i}(t) \dot{\theta}_{\mathrm{ref}}(t)+\left(1-b_{i}(t)\right) \omega_{i}(t),
\end{aligned}
$$

where

$$
\begin{aligned}
b_{i}(t) & =\frac{v_{\mathrm{c}}}{v_{i}(t)} \cos \bar{\theta}_{i}(t) \\
\omega_{i}(t) & =k\left(\alpha_{i}(t)+\arcsin \left(\bar{v} \sin \bar{\theta}_{i}(t)\right)\right),
\end{aligned}
$$

$k>0$ is a positive constant gain, and $\alpha_{i}$ is defined as in (2). We note that such a control law only involves local variables, as it is function of only the measured relative bearing angle and the angle $\bar{\theta}_{i}$, which is an angle difference and therefore independent of the coordinate system.

We will show that this control law brings the autonomous sensors to settle on an equispaced circular formation around a centre moving along the reference direction. As a first step, the following proposition shows an equivalent description of system (1), (6).

Proposition 1: The closed-loop system given by dynamics (1), under the control law (6A)-(6C), is equivalent to the system

$$
\begin{aligned}
\dot{z}_{i}(t) & =v_{\mathrm{c}} \mathrm{e}^{\mathrm{i} \theta_{\text {ref }}(t)}+v_{\mathrm{r}} \mathrm{e}^{\mathrm{i} \gamma_{i}(t)} \\
\dot{\gamma}_{i}(t) & =\omega_{i}(t) .
\end{aligned}
$$

Proof: From (7A) we compute

$$
\left|\dot{z}_{i}\right|^{2}=v_{\mathrm{c}}^{2}+2 v_{\mathrm{c}} v_{\mathrm{r}}\left(\cos \theta_{\mathrm{ref}} \cos \gamma_{i}+\sin \theta_{\mathrm{ref}} \sin \gamma_{i}\right)+v_{\mathrm{r}}^{2},
$$

$$
\arg \dot{z}_{i}=\arctan \frac{v_{\mathrm{c}} \sin \theta_{\mathrm{ref}}+v_{\mathrm{r}} \sin \gamma_{i}}{v_{\mathrm{c}} \cos \theta_{\mathrm{ref}}+v_{\mathrm{r}} \cos \gamma_{i}},
$$

and moreover,

$$
\begin{aligned}
& v_{\mathrm{r}} \cos \gamma_{i}=\left|\dot{z}_{i}\right| \cos \left(\arg \dot{z}_{i}\right)-v_{\mathrm{c}} \cos \theta_{\text {ref }} \\
& v_{\mathrm{r}} \sin \gamma_{i}=\left|\dot{z}_{i}\right| \sin \left(\arg \dot{z}_{i}\right)-v_{\mathrm{c}} \sin \theta_{\text {ref }} .
\end{aligned}
$$


Using (9) we get

$v_{r}\left(\cos \theta_{\text {ref }} \cos \gamma_{i}+\sin \theta_{\text {ref }} \sin \gamma_{i}\right)=\left|\dot{z}_{i}\right| \cos \left(\theta_{\text {ref }}-\arg \dot{z}_{i}\right)-v_{\mathrm{c}}$

and, by substituting it back into (8A), it yields

$$
\left|\dot{z}_{i}\right|^{2}=2 v_{\mathrm{c}} \cos \left(\theta_{\mathrm{ref}}-\arg \dot{z}_{i}\right)\left|\dot{z}_{i}\right|-v_{\mathrm{c}}^{2}+v_{\mathrm{r}}^{2}
$$

Taking the positive solution of this quadratic equation, we obtain the expression for the velocity:

$$
\begin{aligned}
& \left|\dot{z}_{i}\right|=v_{\mathrm{c}} \cos \left(\theta_{\text {ref }}-\arg \dot{z}_{i}\right) \\
& +\sqrt{\left(\cos ^{2}\left(\theta_{\text {ref }}-\arg \dot{z}_{i}\right)-1\right) v_{\mathrm{c}}^{2}+v_{\mathrm{r}}^{2}} \\
& =v_{\mathrm{c}} \cos \left(\theta_{\mathrm{ref}}-\arg \dot{z}_{i}\right)+\sqrt{v_{\mathrm{r}}^{2}-v_{\mathrm{c}}^{2} \sin ^{2}\left(\theta_{\text {ref }}-\arg \dot{z}_{i}\right)} \text {. }
\end{aligned}
$$

Computing the time derivative of (8B) we get

$$
\begin{aligned}
& \frac{\mathrm{d}}{\mathrm{d} t}\left(\arg \dot{z}_{i}\right) \\
& =\frac{v_{\mathrm{c}} \dot{\theta}_{\mathrm{ref}}\left[v_{\mathrm{c}}+v_{\mathrm{r}}\left(\cos \theta_{\mathrm{ref}} \cos \gamma_{i}+\sin \theta_{\mathrm{ref}} \sin \gamma_{i}\right)\right]}{v_{\mathrm{c}}^{2}+2 v_{\mathrm{c}} v_{\mathrm{r}}\left(\cos \theta_{\mathrm{ref}} \cos \gamma_{i}+\sin \theta_{\text {ref }} \sin \gamma_{i}\right)+v_{\mathrm{r}}^{2}} \\
& \quad+\frac{v_{\mathrm{r}} \dot{\gamma}_{i}\left[v_{\mathrm{r}}+v_{\mathrm{c}}\left(\cos \theta_{\mathrm{ref}} \cos \gamma_{i}+\sin \theta_{\mathrm{ref}} \sin \gamma_{i}\right)\right]}{v_{\mathrm{c}}^{2}+2 v_{\mathrm{c}} v_{\mathrm{r}}\left(\cos \theta_{\text {ref }} \cos \gamma_{i}+\sin \theta_{\text {ref }} \sin \gamma_{i}\right)+v_{\mathrm{r}}^{2}}
\end{aligned}
$$

substituting again (10):

$$
\begin{aligned}
\frac{\mathrm{d}}{\mathrm{d} t}\left(\arg \dot{z}_{i}\right)= & \frac{v_{\mathrm{c}}\left|\dot{z}_{i}\right| \cos \left(\theta_{\mathrm{ref}}-\arg \dot{z}_{i}\right) \dot{\theta}_{\mathrm{ref}}}{2 v_{\mathrm{c}}\left|\dot{z}_{i}\right| \cos \left(\theta_{\mathrm{ref}}-\arg \dot{z}_{i}\right)-v_{\mathrm{c}}^{2}+v_{\mathrm{r}}^{2}} \\
& +\frac{\left(v_{\mathrm{r}}^{2}+v_{\mathrm{c}}\left|\dot{z}_{i}\right| \cos \left(\theta_{\mathrm{ref}}-\arg \dot{z}_{i}\right)-v_{\mathrm{c}}^{2}\right) \dot{\gamma}_{i}}{2 v_{\mathrm{c}}\left|\dot{z}_{i}\right| \cos \left(\theta_{\text {ref }}-\arg \dot{z}_{i}\right)-v_{\mathrm{c}}^{2}+v_{\mathrm{r}}^{2}}
\end{aligned}
$$

and, finally, by (11),

$$
\begin{aligned}
\frac{\mathrm{d}}{\mathrm{d} t}\left(\arg \dot{z}_{i}\right)=\frac{v_{\mathrm{c}} \cos \left(\theta_{\text {ref }}-\arg \dot{z}_{i}\right)}{\left|\dot{z}_{i}\right|} \dot{\theta}_{\text {ref }} \\
+\left(1-\frac{v_{\mathrm{c}} \cos \left(\theta_{\text {ref }}-\arg \dot{z}_{i}\right)}{\left|\dot{z}_{i}\right|}\right) \dot{\gamma}_{i} .
\end{aligned}
$$

We can notice that, with notation $v_{i}=\left|\dot{z}_{i}\right|$ and $\theta_{i}=\arg \dot{z}_{i}$ as in (1), the final expressions of equations (12) and (13) correspond to our choice of control input $\boldsymbol{u}_{i}=\left[v_{i}, \dot{\theta}_{i}\right]^{T}$ in $(6 \mathrm{~A})$ and $(6 \mathrm{~B})$, thus showing the equivalence of systems (1), (6A)-(6C) and (7).

Remark 1: We can see from this equivalence that the dynamics of each sensor is given by the superposition of a motion with speed $v_{\mathrm{c}}$ in the common direction $\theta_{\text {ref }}$ and a motion with speed $v_{\mathrm{r}}$ and heading $\gamma_{i}$ such that $\dot{\gamma}_{i}=\omega_{i}$. In particular, since the direction $\theta_{\text {ref }}$ is common to each autonomous sensor, the motion with speed $v_{\mathrm{c}}$ results in a translation of the whole formation in such direction. We need to show that our law ensures that the sensors settle on a circular equispaced formation (thus allowing for a gradient estimation based on the Poisson integral formula), i.e., they asymptotically tend to a formation in which each sensor has position and velocity

$$
\begin{aligned}
& z_{i}(t)=z_{\mathrm{c}}(t)+r \mathrm{e}^{\mathrm{i} \zeta_{i}(t)} \\
& \dot{z}_{i}(t)=v_{\mathrm{c}} \mathrm{e}^{\mathrm{i} \theta_{\text {ref }}(t)}+v_{\mathrm{r}} \mathrm{e}^{\mathrm{i} \gamma_{i}(t)},
\end{aligned}
$$

with $\zeta_{i}(t)=\zeta_{0}(t)+\frac{2 \pi}{n} i$ and $\gamma_{i}(t)=\zeta_{i}(t)+\frac{\pi}{2}$.

\section{Convergence to formation}

Here, we show how the control law (6) can bring the sensors to the desired circular formation. We start by recalling some results by Marshall et al. [16].

Let us consider a fleet of autonomous underactuated vehicles described by the dynamics (1). By a suitable change of coordinates [16], the system (1) can be described by the new state variables

$$
\begin{aligned}
d_{i}(t) & =\left|z_{i+1}(t)-z_{i}(t)\right| \\
\alpha_{i}(t) & =\arg \left(z_{i+1}(t)-z_{i}(t)\right)-\theta_{i}(t) \\
\beta_{i}(t) & =\theta_{i}(t)-\theta_{i+1}(t)-\pi .
\end{aligned}
$$

With such state variables we lose any information about the absolute position of each vehicle; nonetheless, they are more suitable to describe the formation reached, as they define the position of each vehicle in terms of relative variables with respect to each other's neighbours, such as the relative distance $d_{i}$ and the relative bearing angle $\alpha_{i}$. For the subsequent analysis, it is important to notice that the above-defined variables satisfy the following constraints ${ }^{1}$ :

$$
\begin{aligned}
& \sum_{i=1}^{n} \beta_{i}=-n \pi \\
& \sum_{i=1}^{n} d_{i} \mathrm{e}^{\mathrm{i}\left[\alpha_{i}-(i-1) \pi-\sum_{j=1}^{i-1} \beta_{j}\right]}=0 .
\end{aligned}
$$

Eq. (15) is immediate from the definition of $\beta_{i}$, while Eq. (16) is obtained from $\sum_{i=1}^{n}\left(z_{i+1}-z_{i}\right)=0$, where the vector $z_{i+1}-$ $z_{i}$ can be expressed with its modulus $d_{i}$ and its argument $\arg \left(z_{i+1}-z_{i}\right)=\alpha_{i}+\theta_{i}=\alpha_{i}+\theta_{1}-\sum_{j=1}^{i-1}\left(\beta_{j}+\pi\right)$.

The authors in [16] propose the following control law

$$
v_{i}(t)=\tilde{v} \quad \dot{\theta}_{i}(t)=k \alpha_{i}(t)
$$

with $\tilde{v}, k>0$ constant parameters, and show that the closedloop dynamics, in the state variables (14), becomes

$$
\begin{aligned}
& \dot{d}_{i}(t)=-\tilde{v}\left(\cos \alpha_{i}(t)+\cos \left(\alpha_{i}(t)+\beta_{i}(t)\right)\right) \\
& \dot{\alpha}_{i}(t)=\frac{\tilde{v}\left(\sin \alpha_{i}(t)+\sin \left(\alpha_{i}(t)+\beta_{i}(t)\right)\right)}{d_{i}(t)}-k \alpha_{i}(t) \\
& \dot{\beta}_{i}(t)=k\left(\alpha_{i}(t)-\alpha_{i+1}(t)\right) .
\end{aligned}
$$

Their study shows that the equilibrium points of such dynamics correspond to the vehicles disposed as the vertices of an ordinary regular polygon, and thus being equispatially disposed on a circle (whose radius is given by $r=\frac{n \tilde{v}}{k \pi}$ ); moreover, they analyze stability of such equilibria. The following proposition summarizes some of their most important findings, while in the remainder of this section we will show how such results can be applied to our problem formulation.

Proposition 2 ([16, Lemma 2, Thm. 5 and Coroll. 4]): The system (18), restricted to the invariant manifold $\mathcal{M} \subset \mathbb{R}^{3 n}$ described by the constraints in (15)-(16), has $2(n-1)$ equilibria, among which the two following ones:

$$
\bar{d}_{i}=\bar{d}=\frac{2 \tilde{v}}{k \bar{\alpha}} \sin \bar{\alpha}
$$

\footnotetext{
${ }^{1}$ We use the notational convention that a sum over an empty set of indices is equal to zero, namely, $\sum_{j=1}^{0} \beta_{j}=0$
} 


$$
\begin{aligned}
\bar{\alpha}_{i} & =\bar{\alpha}= \pm \frac{\pi}{n} \\
\bar{\beta}_{i} & =\bar{\beta}=\pi-2 \bar{\alpha} .
\end{aligned}
$$

The two above-described equilibria are locally asymptotically stable.

The interested reader can find the proof in [16]; it is based on geometrical considerations to characterize equilibria, on linearization around equilibria of the system restricted to the invariant manifold, and on a careful study of the eigenvalues of the resulting linear system.

Since our control law is different from the one proposed in [16], also our closed-loop dynamics will differ from (18), and the results of Theorem 2 do not apply directly. Nevertheless, defining the variables

$$
\begin{aligned}
& \tilde{\alpha}_{i}(t)=\arg \left(z_{i+1}(t)-z_{i}(t)\right)-\gamma_{i}(t) \\
& \tilde{\beta}_{i}(t)=\gamma_{i}(t)-\gamma_{i+1}(t)-\pi,
\end{aligned}
$$

with $\gamma_{i}$ as in (9), we will show that in the new state $d_{i}(t), \quad \tilde{\alpha}_{i}(t), \tilde{\beta}_{i}(t)$ our closed-loop system converges to the same equilibrium points as (19).

First, we notice that the above-defined variables satisfy the same constraints as the variables in (14), namely

$$
\begin{aligned}
& \sum_{i=1}^{n} \tilde{\beta}_{i}=-n \pi \\
& \sum_{i=1}^{n} \mathrm{~d}_{i} \mathrm{e}^{\mathrm{i}\left[\tilde{\alpha}_{i}-(i-1) \pi-\sum_{j=1}^{i-1} \tilde{\beta}_{j}\right]}=0 .
\end{aligned}
$$

Then, in the following, we prove that, under the control law (6), the variables $d_{i}, \tilde{\alpha}_{i}, \tilde{\beta}_{i}$ defined in (14A) and (20) have the same dynamics as (18), so that the same equilibrium analysis applies.

Proposition 3: For the system (7), with $\omega_{i}$ as in (6D), the dynamics in the state variables (14A), (20) is

$$
\begin{aligned}
& \dot{d}_{i}(t)=-v_{\mathrm{r}}\left(\cos \tilde{\alpha}_{i}(t)+\cos \left(\tilde{\alpha}_{i}(t)+\tilde{\beta}_{i}(t)\right)\right) \\
& \dot{\tilde{\alpha}}_{i}(t)=\frac{v_{\mathrm{r}}\left(\sin \tilde{\alpha}_{i}(t)+\sin \left(\tilde{\alpha}_{i}(t)+\tilde{\beta}_{i}(t)\right)\right)}{d_{i}(t)}-k \tilde{\alpha}_{i}(t) \\
& \dot{\tilde{\beta}}_{i}(t)=k\left(\tilde{\alpha}_{i}(t)-\tilde{\alpha}_{i+1}(t)\right)
\end{aligned}
$$

Proof: We start by showing that the control law (7B) is equivalent to the control law $\dot{\gamma}_{i}=k \tilde{\alpha}_{i}$. We define

$$
\delta_{i}(t)=\theta_{i}(t)-\gamma_{i}(t) .
$$

Taking the sine and using (9), we obtain

$$
\begin{aligned}
& \sin \delta_{i}= \sin \theta_{i} \frac{v_{i} \cos \theta_{i}-v_{\mathrm{c}} \cos \theta_{\mathrm{ref}}}{v_{\mathrm{r}}} \\
&-\cos \theta_{i} \frac{v_{i} \sin \theta_{i}-v_{\mathrm{c}} \sin \theta_{\mathrm{ref}}}{v_{\mathrm{r}}} \\
&=\frac{v_{\mathrm{c}}}{v_{\mathrm{r}}}\left(\cos \theta_{i} \sin \theta_{\text {ref }}-\sin \theta_{i} \cos \theta_{\text {ref }}\right)=\bar{v} \sin \bar{\theta}_{i},
\end{aligned}
$$

from which it follows that $\delta_{i}(t)=\arcsin \left(\bar{v} \sin \bar{\theta}_{i}(t)\right)$. Therefore, we have that

$\dot{\gamma}_{i}=k\left(\alpha_{i}+\delta_{i}\right)=k\left(\arg \left(z_{i+1}-z_{i}\right)-\theta_{i}+\theta_{i}-\gamma_{i}\right)=k \tilde{\alpha}_{i}$.

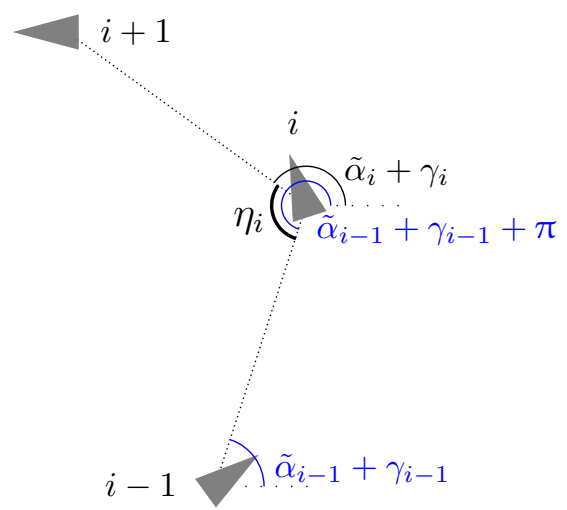

Fig. 4: The internal angle $\eta_{i}$ between consecutive sensors.

Now we have

$$
\begin{aligned}
& \dot{z}_{i}(t)=v_{\mathrm{c}} \mathrm{e}^{\mathrm{i} \theta_{\text {ref }}(t)}+v_{\mathrm{r}} \mathrm{e}^{\mathrm{i} \gamma_{i}(t)} \\
& \dot{\gamma}_{i}(t)=k \tilde{\alpha}_{i}(t) .
\end{aligned}
$$

From this system, the dynamics (23) are obtained, similarly to the fact that system (1), (17) gives dynamics (18) in [16] (full details of the calculations can be found in the proof of Proposition 5.3 in [17]).

Proposition 4: The system (14), restricted to the invariant manifold $\mathcal{M} \subset \mathbb{R}^{3 n}$ described by the constraints in (21)-(22), has $2(n-1)$ equilibria, among which the two following ones:

$$
\begin{aligned}
& \bar{d}_{i}=\bar{d}=\frac{2 v_{\mathrm{r}}}{k \bar{\alpha}} \sin \bar{\alpha} \\
& \overline{\tilde{\alpha}}_{i}=\bar{\alpha}= \pm \frac{\pi}{n} \\
& \overline{\tilde{\beta}}_{i}=\bar{\beta}=\pi-2 \bar{\alpha} .
\end{aligned}
$$

The two above-described equilibria are locally asymptotically stable.

Proof: By Prop. 3 the system $d_{i}(t), \tilde{\alpha}_{i}(t), \tilde{\beta}_{i}(t)$ has dynamics equivalent to (18). Hence, applying Prop. 2 yields to the equilibria (25).

Proposition 5: At the equilibria (25), the relative positions $z_{i+1}-z_{i}$ draw an ordinary regular polygon.

Proof: The internal angle between sensors $z_{i-1}, z_{i}, z_{i+1}$ (see Fig. 4) can be expressed as

$$
\begin{aligned}
\eta_{i}(t) & =\arg \left(z_{i}(t)-z_{i-1}(t)\right)+\pi-\arg \left(z_{i+1}(t)-z_{i}(t)\right) \\
& =\tilde{\alpha}_{i-1}(t)+\gamma_{i-1}(t)+\pi-\tilde{\alpha}_{i}(t)-\gamma_{i}(t) \\
& =\tilde{\alpha}_{i-1}(t)-\tilde{\beta}_{i-1}(t)-\tilde{\alpha}_{i}(t)
\end{aligned}
$$

at the equilibrium, $\tilde{\alpha}_{i}=\bar{\alpha}$ and $\tilde{\beta}_{i}=\bar{\beta}, \forall i$, gives

$$
\eta_{i}(t)=-\bar{\beta}, \forall i \text {. }
$$

Hence, the relative positions at the equilibrium are both equilateral (because $\bar{d}_{i}=\bar{d} \forall i$ ) and equiangular, and thus represent an ordinary regular polygon.

\section{Gradient-Ascent Motion of the Formation Centroid}

Our goal is to use the sensors measurements to compute a discrete approximation of the gradient formula (5), and then to perform a gradient ascent using the (approximated) 
gradient argument as reference $\theta_{\text {ref }}$ for the control law (6). The computation of the gradient approximation will be discussed in Section IV, while here we discuss how the control law (6) indeed drives the centroid of the formation to follow the reference direction and perform gradient ascent towards the source. We will make use of the result of Prop.s 4 and 5, which ensure local asymptotic convergence to an equispaced circular formation, and we will study the motion of the formation centroid.

Proposition 6: If the initial conditions of the agents are such that the corresponding system (23) is in the attractive basin of one of the two locally asymptotically stable equilibria (25), then

$$
\arg \dot{z}_{\mathrm{c}}(t)-\theta_{\text {ref }}(t) \rightarrow 0 .
$$

Proof: The dynamics of the formation centroid (i.e., the barycentre of positions $z_{i}$ 's) is given by

$$
\dot{z}_{\mathrm{c}}(t)=\frac{1}{n} \sum_{i=1}^{n} \dot{z}_{i}(t)=v_{\mathrm{c}} \mathrm{e}^{\mathrm{i} \theta_{\text {ref }}(t)}+\frac{v_{\mathrm{r}}}{n} \sum_{i=1}^{n} \mathrm{e}^{\mathrm{i} \gamma_{i}(t)} .
$$

We can easily show that, at any of the equilibria (25),

$$
\dot{z}_{\mathrm{c}}(t)=v_{\mathrm{c}} \mathrm{e}^{\mathrm{i} \theta_{\text {ref }}(t)} .
$$

Indeed, the result of Prop. 5 implies that, at equilibria (25), they occupy positions $z_{i}$ which are equispaced along a circle. In particular, Eq. (20B) implies that $\gamma_{i+1}-\gamma_{i}=-\tilde{\beta}_{i}-\pi \forall i$ which, at the equilibria (25), gives $\gamma_{i}=\gamma_{0}+\frac{2 \pi}{n} i$ for all $i$, and hence $\sum_{i=1}^{n} \mathrm{e}^{\mathrm{i} \gamma_{i}(t)}=0$.

Then, the local asymptotic stability of such equilibria ensures the asymptotic convergence of $\dot{z}_{\mathrm{c}}(t)-v_{\mathrm{c}} \mathrm{e}^{\mathrm{i} \theta_{\text {ref }}(t)}=$ $\frac{v_{\mathrm{r}}}{n} \sum_{i=1}^{n} \mathrm{e}^{\mathrm{i} \gamma_{i}(t)}$ to zero, and hence also ensures that $\arg \dot{z}_{\mathrm{c}}(t)-$ $\theta_{\text {ref }}^{n}(t) \rightarrow 0$.

This proposition means that, if the agents have initial positions such that they will converge to circular formation, then, while this convergence happens, their centroid will follow with increasing precision the reference direction. In particular, given any $\varepsilon>0$, there exists a time $\bar{t}$ such that, for all $t \geq \bar{t}$, $\left|\arg \dot{z}_{\mathrm{c}}(t)-\theta_{\text {ref }}(t)\right| \leq \varepsilon$; moreover, velocities of individual agents and of the formation centroid are all bounded by $v_{\mathrm{c}}+v_{\mathrm{r}}$, so that there is no risk of divergence in finite time.

In the next section we will discuss how to compute a reference $\theta_{\text {ref }}(t)$ which is an approximation of the argument of the gradient $\nabla f\left(z_{\mathrm{c}}(t)\right)$ (at least for sufficiently large times, when circular formation is nearly attained). Now, let us assume that such a reference is given, and that for all $t \geq \tilde{t}$, $\left|\theta_{\text {ref }}(t)-\arg \nabla f\left(z_{\mathrm{c}}(t)\right)\right| \leq \tilde{\varepsilon}$ for some time $\tilde{t}$ and for some error bound $\tilde{\varepsilon}<\frac{\pi}{2}$. Then, we can clearly find $\varepsilon$ such that $\varepsilon+\tilde{\varepsilon}<\frac{\pi}{2}$, and a time $\bar{t} \geq \tilde{t}$ such that, for all $t \geq \bar{t}$,

$$
\left|\arg \dot{z}_{\mathrm{c}}(t)-\arg \nabla f\left(z_{\mathrm{c}}(t)\right)\right| \leq \varepsilon+\tilde{\varepsilon}<\frac{\pi}{2} .
$$

This inequality ensures that the formation centroid performs gradient ascent, namely that $f\left(z_{\mathrm{c}}(t)\right)$ increases along trajectories; indeed, the derivative of $f$ along trajectories of $z_{\mathrm{c}}(t)$ is given by the scalar product $\nabla f\left(z_{\mathrm{c}}(t)\right) \cdot \dot{z}_{\mathrm{c}}(t)$, which is positive since $\left|\arg \dot{z}_{\mathrm{c}}(t)-\arg \nabla f\left(z_{\mathrm{c}}(t)\right)\right|<\frac{\pi}{2}$.

Gradient ascent is usually applied to convex functions, while here we are considering harmonic functions, which might not be convex. However, the local maximum principle ensures that $f$ does not have any local maximum inside $\Omega$ : hence, gradient ascent search is ensured not to get trapped in any local maximum, except possibly on the outer boundary $\partial \tilde{\Omega}$; some simple rule can be introduced that allows to distinguish the external boundary from the source, e.g., some rough bound on the source intensity.

\section{IMPLEMENTATION DETAILS}

We discuss here how to implement the control law proposed in Sect. III. We assume to have no absolute position information, and very little relative one: this means that the sensors neither know their absolute position, nor their relative one with respect to the formation centroid or to each other, and they do not know their absolute orientation $\theta_{i}$ either. They are only able to measure the pointwise value of the diffusing quantity at their position, i.e., $f\left(z_{i}(t)\right)$, and the relative bearing angle with respect to their neighbour $\alpha_{i}$; in addition, they know the common parameters $v_{\mathrm{c}}, v_{\mathrm{r}}, k$ and their number $n$.

To drive the sensors to the source location, we make use of the control input defined in (6), which involves the difference $\bar{\theta}_{i}$ between the reference input $\theta_{\text {ref }}$ and the current heading of the sensor $\theta_{i}$, and where we want the reference $\theta_{\text {ref }}$ to be the angle of the approximation, computed by each sensor $i$, of the gradient at the formation centroid. To obtain such an approximation we propose a discrete-time algorithm, involving an exchange of messages among the agents, which is run within every sampling time; the value of the gradient approximation $g_{i}(t)$, and consequently of its argument, is then held constant until the next value is available. The algorithm is designed to use the information collected by each sensor and produce, in a distributed way, a discrete approximation of the integral formula (5), i.e., to compute the Riemann sum

$$
\widehat{\nabla f}\left(z_{\mathrm{c}}(t)\right)=\frac{2}{n r^{2}} \sum_{i=1}^{n} f\left(z_{i}(t)\right)\left(z_{i}(t)-z_{\mathrm{c}}(t)\right),
$$

and then obtain the difference $\bar{\theta}_{i}=\theta_{\text {ref }}-\theta_{i}$, where ideally $\theta_{\text {ref }}=\arg \widehat{\nabla f}$. The difficulty lies in the fact that the vector $z_{i}-z_{\mathrm{c}}$ is not directly available, as we assumed not to have any position information. We propose the following technique.

During every time interval $(t, t+\Delta t)$, with $\Delta t$ sufficiently long, each sensor $i$ performs $n-1$ iterations which involve an exchange of messages with its preceding and following neighbours: each sensor $i$ receives the current value computed by its predecessors, rotates it of an angle $\kappa= \pm \frac{2 \pi}{n}$ (where the sign depends on the direction of the rotation of the agents at the equilibrium), adds its own measurement $f\left(z_{i}\right)$ and sends the result to its successor.

In the sequel we will suppose for simplicity to be in the attractive region of the equilibrium with $\bar{\alpha}>0$ (i.e., a counterclockwise rotation), hence, we will use a rotation angle of $\kappa=-\frac{2 \pi}{n}$. A possible more general implementation can use a rotation angle depending on the current relative bearing angle measured, i.e., $\kappa_{i}(t)=-\operatorname{sign}\left(\alpha_{i}(t)\right) \frac{2 \pi}{n}$.

The argument of the vector obtained in this way is the $i$ th reference, which is then used to compute the difference $\bar{\theta}_{i}$ and then, together with the relative bearing angle $\alpha_{i}$ which each 
sensor $i$ measure with respect to its follower, the new control $\boldsymbol{u}_{i}$. The detailed algorithm is reported below.

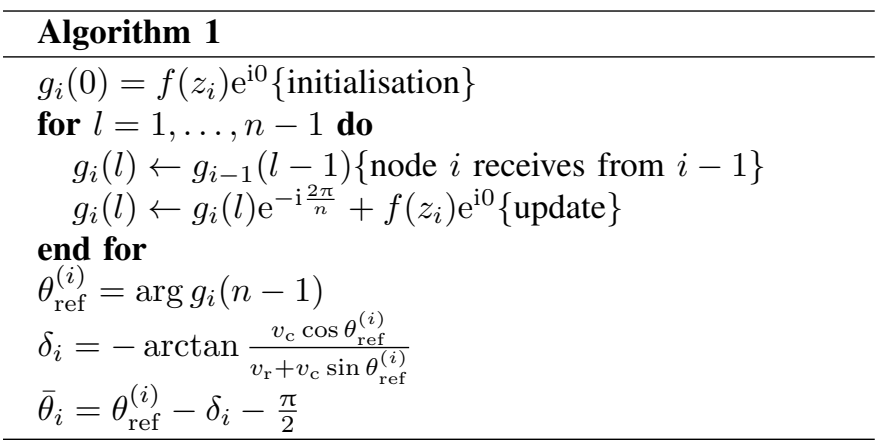

The following proposition shows the effectiveness of the proposed algorithm if the sensors are already at the equilibrium.

Proposition 7: If the $n$ sensors described by the dynamics (23) are at the equilibrium (25) with $\bar{\alpha}>0$, then $\bar{\theta}_{i}$ computed with Algorithm 1 corresponds to the difference

$$
\bar{\theta}_{i}=\arg \widehat{\nabla f}\left(z_{\mathrm{c}}\right)-\theta_{i} \text {. }
$$

Proof: When the sensors are at the equilibrium (25) with $\bar{\alpha}>0$, they are turning equispaced in circle around their centroid $z_{\mathrm{c}}$; hence we have that

$$
\gamma_{i}=\left\{\begin{array}{l}
\arg \left(z_{i}-z_{\mathrm{c}}\right)+\frac{\pi}{2} \\
\gamma_{0}+\frac{2 \pi}{n} i
\end{array} \quad, \forall i\right.
$$

in particular, the second one implies that $\gamma_{j}=\gamma_{i}+\frac{2 \pi}{n}(j-i)$ for all $i, j$. Using these properties, we can rewrite the argument of the Riemann sum in (27) as

$$
\begin{aligned}
& \arg \widehat{\nabla f}\left(z_{\mathrm{c}}\right)=\arg \sum_{j=1}^{n} f\left(z_{j}\right) \mathrm{e}^{\mathrm{i}\left(\gamma_{j}-\frac{\pi}{2}\right)} \\
& \quad=\arg \sum_{j=1}^{n} f\left(z_{j}\right) \mathrm{e}^{\mathrm{i}\left[\gamma_{i}+\frac{2 \pi}{n}(j-i)-\frac{\pi}{2}\right]}, \forall i .
\end{aligned}
$$

Node $i$, at the end of the $n-1$ iterations in Algorithm 1, has computed

$$
g_{i}(n-1)=\sum_{j=1}^{n} f\left(z_{j}\right) \mathrm{e}^{\mathrm{i} \frac{2 \pi}{n}(j-i)},
$$

so that

$$
\theta_{\text {ref }}^{(i)}=\arg g_{i}(n-1)=\arg \widehat{\nabla f}\left(z_{\mathrm{c}}\right)-\gamma_{i}+\frac{\pi}{2} .
$$

We want to show that the solution of system

$$
\begin{aligned}
& \delta_{i}=-\arctan \frac{v_{\mathrm{c}} \cos \theta_{\mathrm{ref}}^{(i)}}{v_{\mathrm{r}}+v_{\mathrm{c}} \sin \theta_{\mathrm{ref}}^{(i)}} \\
& \bar{\theta}_{i}=\theta_{\text {ref }}^{(i)}-\delta_{i}-\frac{\pi}{2}
\end{aligned}
$$

and the one of

$$
\begin{aligned}
& \delta_{i}=\theta_{i}-\gamma_{i} \\
& \bar{\theta}_{i}=\arg \widehat{\nabla f}\left(z_{\mathrm{c}}\right)-\theta_{i}
\end{aligned}
$$

coincide, thus showing that system (31) used in the algorithm correctly computes the quantities of interest defined by (32).
We already know from (24) that $\delta_{i}=\theta_{i}-\gamma_{i}$ implies $\sin \delta_{i}=$ $\bar{v} \sin \bar{\theta}_{i}$; thus, combining it with (30), system (32) becomes

$$
\begin{aligned}
\sin \delta_{i} & =\bar{v} \sin \bar{\theta}_{i} \\
\bar{\theta}_{i} & =\theta_{\text {ref }}^{(i)}+\gamma_{i}-\frac{\pi}{2}-\theta_{i}=\theta_{\text {ref }}^{(i)}-\delta_{i}-\frac{\pi}{2} .
\end{aligned}
$$

Solving for $\sin \bar{\theta}_{i}$ we get

$$
\sin \bar{\theta}_{i}=\left\{\begin{array}{l}
\frac{\sin \delta_{i}}{\bar{v}} \\
-\cos \theta_{\text {ref }}^{(i)} \cos \delta_{i}-\sin \theta_{\text {ref }}^{(i)} \sin \delta_{i} ;
\end{array}\right.
$$

equating the solutions and multiplying by $\frac{\bar{v}}{\cos \delta_{i}}$ yields

$$
\tan \delta_{i}=-\frac{\bar{v} \cos \theta_{\mathrm{ref}}^{(i)}}{1+\bar{v} \sin \theta_{\mathrm{ref}}^{(i)}},
$$

and assuming that $\delta_{i} \in\left[-\frac{\pi}{2}, \frac{\pi}{2}\right]$ and $\bar{\theta}_{i} \in[-\pi, \pi]$ we obtain

$$
\delta_{i}=-\arctan \frac{v_{\mathrm{c}} \cos \theta_{\mathrm{ref}}^{(i)}}{v_{\mathrm{r}}+v_{\mathrm{c}} \sin \theta_{\mathrm{ref}}^{(i)}} .
$$

\section{Guaranteeing a common reference}

As one may notice, Algorithm 1 gives the argument of the correct gradient approximation only when the sensors are in formation. Otherwise, not only $\theta_{\text {ref }}^{(i)}$ is not ensured to be a good approximation of the gradient argument, but it is not even a common reference, i.e., $\bar{\theta}_{i}(t)+\theta_{i}(t)$ is not the same for all $i$ (while it should be equal to a common reference in order to apply Prop. 1). Indeed, Eq. (28) holds only at the formation. This contradicts the assumption we made about the reference $\theta_{\text {ref }}$ being the same for every sensor, which guarantees the convergence to the equilibria (25) and thus to a circular equispaced formation; nevertheless, we proposed this implementation for its simplicity, since it shows a good behaviour in simulations, as we will show in Section V.

Below, we propose an alternative algorithm which ensures that all sensors have a common reference $\theta_{\text {ref }}$, regardless their relative positions and orientations.

To do so, we need to designate a leader sensor, say node 1 . Moreover, we need the measure of the relative bearing angle with respect to the predecessor; i.e., in addition to the alreadydefined angle $\alpha_{i}$, we assume that each sensor $i$ can also measure the angle

$$
\alpha_{i}^{-}(t)=\arg \left(z_{i-1}(t)-z_{i}(t)\right)-\theta_{i}(t)
$$

The idea is to perform, after a first phase as in Algorithm 1, a second communication round enforcing $\bar{\theta}_{i}=\theta_{\text {ref }}-\theta_{i}$ for every $i$, with $\theta_{\text {ref }}=\bar{\theta}_{1}+\theta_{1}$.

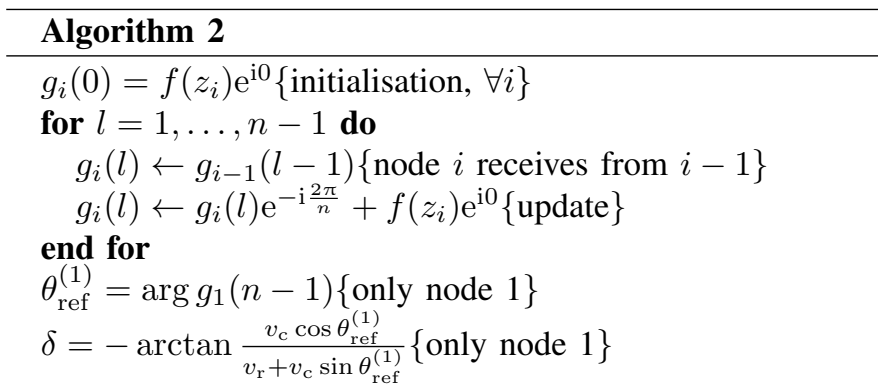




$$
\begin{aligned}
& \bar{\theta}_{1}=\theta_{\text {ref }}^{(1)}-\delta-\frac{\pi}{2}\{\text { only node } 1\} \\
& \text { for } i=1, \ldots, n-1 \text { do } \\
& \iota=\bar{\theta}_{i}-\alpha_{i}-\frac{\pi}{2}\{\text { node } i\} \\
& \text { node } i+1 \text { receives } \iota \text { from } i \\
& \bar{\theta}_{i+1}=\iota+\alpha_{i+1}^{-}-\frac{\pi}{2}\{\text { node } i+1\} \\
& \text { end for }
\end{aligned}
$$

Proposition 8: If each sensor $i$ computes $\bar{\theta}_{i}$ as in Algorithm 2 , then $\bar{\theta}_{i}(t)=\theta_{\text {ref }}(t)-\theta_{i}(t) \forall i$, where $\theta_{\text {ref }}=\bar{\theta}_{1}+\theta_{1}$, and $\bar{\theta}_{1}$ is the same as in Algorithm 1 .

Proof: It is easy to see that, with Algorithm 2, $\bar{\theta}_{1}$ is the same as in Algorithm 1, and

$$
\begin{aligned}
\bar{\theta}_{i+1} & =\bar{\theta}_{i}+\alpha_{i+1}^{-}-\alpha_{i}-\pi \\
& =\bar{\theta}_{i}+\arg \left(z_{i}-z_{i+1}\right)-\theta_{i+1}-\arg \left(z_{i+1}-z_{i}\right)+\theta_{i}-\pi \\
& =\bar{\theta}_{i}+\theta_{i}-\theta_{i+1},
\end{aligned}
$$

from which, recursively, we get $\bar{\theta}_{i}=\bar{\theta}_{1}+\theta_{1}-\theta_{i}$.

\section{Alternative communication strategies}

Algorithms 1 and 2 are based on a ring communication topology, namely messages are sent from sensor $i$ to $i+1$, but they can be easily adapted to a variety of other communication setups, by making use of well-known linear average-consensus algorithms. Consider a given communication graph describing which sensors are allowed to send messages to one another, and a linear consensus algorithm, described by $n \times n$ matrices $P(0), P(1), \ldots$, where $P_{i j}$ is 0 if there is no edge from $j$ to $i$. At each iteration of the consensus algorithm, each node i sends its current state, say $\xi_{i}(l)$, to its neighbors, and then computes the state update $\xi_{i}(l+1)=\sum_{j} P_{i j}(l) \xi_{j}(l)$, using the received neighbors states and its own state. Under mild assumptions on the communication graph, there are well-known constructions of asymptotic average consensus algorithms, such that $\lim _{l \rightarrow \infty} \xi(l)=\frac{1}{n} \sum_{j} \xi_{j}(0)$ (e.g., with time-invariant doubly-stochastic $P$ [18]), of finite-time average consensus algorithms, such that $\xi(L)=\frac{1}{n} \sum_{j} \xi_{j}(0)$ for some $L$ [19], and gossip consensus, where $P(k)$ 's are random matrices consistent with random asynchronous communications while ensuring some stochastic convergence near the average of initial values [20]. Algorithm 1 can be modified as follows, where we use a given linear consensus algorithm, but we rotate state vectors at each iteration in a suitable way.

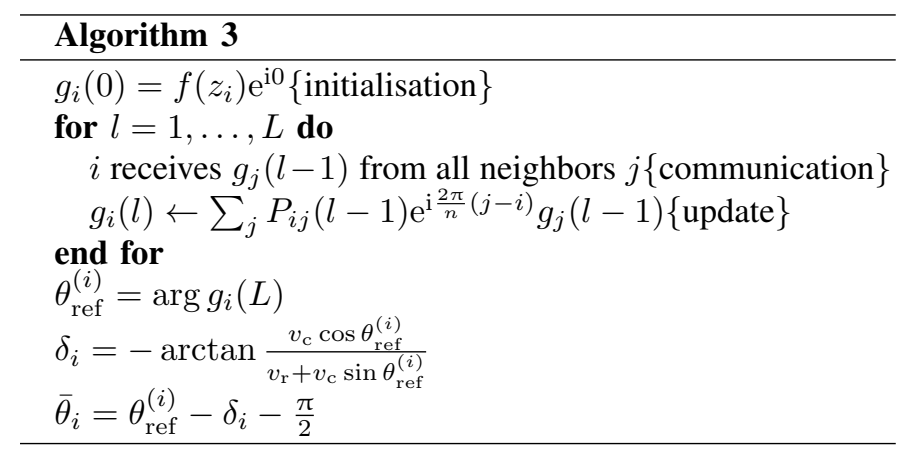

At the end of the cycle of communications and updates, we get

$g_{i}(L)=\sum_{j_{L-1}} \cdots \sum_{j_{0}} P_{i j_{L-1}}(L-1) \cdot \cdots \cdot P_{j_{1} j_{0}}(0) \mathrm{e}^{\mathrm{i}\left(j_{0}-i\right)} f\left(z_{j_{0}}\right)$.
If we define $\xi_{j}(0)=f\left(z_{j}\right) \mathrm{e}^{\mathrm{i} \frac{2 \pi}{n} j}$, the standard linear consensus (with no rotation) would give

$\xi_{i}(L)=\sum_{j_{L-1}} \cdots \sum_{j_{0}} P_{i j_{L-1}}(L-1) \cdot \ldots \cdot P_{j_{1} j_{0}}(0) f\left(z_{j_{0}}\right) \mathrm{e}^{\mathrm{i} \frac{2 \pi}{n} j_{0}}$.

Hence, $g_{i}(L)=\mathrm{e}^{\mathrm{i}\left(-\frac{2 \pi}{n} i\right)} \xi_{i}(L)$, and inherits the convergence properties of the chosen consensus algorithm: if this is a finitetime average consensus, we get

$$
g_{i}(L)=\mathrm{e}^{\mathrm{i}\left(-\frac{2 \pi}{n} i\right)} \frac{1}{n} \sum_{j} \xi_{j}(0)=\frac{1}{n} \sum_{j} f\left(z_{j}\right) \mathrm{e}^{\mathrm{i} \frac{2 \pi}{n}(j-i)} .
$$

Comparing this with (29), we can see that the only difference with Algorithm 1 is a factor $\frac{1}{n}$, which is irrelevant when computing the argument $\theta_{\text {ref }}^{(i)}=\arg g_{i}(L)$, so that the result of Algorithm 3 is exactly the same as the one of Algorithm 1. When consensus only has asymptotic convergence, the same applies to Algorithm 3, which then gives an approximation of the value obtained in Algorithm 1.

In a similar way, also Algorithm 2 can be adapted, provided that each node can measure the relative angle $\alpha_{i j}=\arg \left(z_{j}-\right.$ $\left.z_{i}\right)-\theta_{i}$ with respect to all of its in- and out-neighbors. The first part, to find $\bar{\theta}_{1}$, follows the same technique as in Algorithm 3. Then, node 1 initializes its state to $\chi_{1}(0)=\mathrm{e}^{\mathrm{i} \bar{\theta}_{1}}$, while all other nodes set $\chi_{i}(0)=0$. Again, a modified consensus is run, where messages are suitably rotated versions of the local states: at iteration $l$, node $i$ receives $\mathrm{e}^{\mathrm{i}\left(-\alpha_{j i}-\frac{\pi}{2}\right)} \chi_{j}(l-1)$ from each neighbor $j$, and then computes

$$
\chi_{i}(l)=\sum_{j} P_{i j}(l-1) \mathrm{e}^{\mathrm{i}\left(\alpha_{i j}-\frac{\pi}{2}\right)} \mathrm{e}^{\mathrm{i}\left(-\alpha_{j i}-\frac{\pi}{2}\right)} \chi_{j}(l-1) .
$$

After $L$ iterations, each node $i$ computes $\bar{\theta}_{i}=\arg \chi_{i}(L)$, thus ending the algorithm.

It is easy to show that, with finite-time average consensus, $\chi_{i}(L)=\frac{1}{n} \mathrm{e}^{\mathrm{i}\left(\bar{\theta}_{1}+\theta_{1}-\theta_{i}\right)}$, so that its argument gives exactly the same $\bar{\theta}_{i}$ as in Algorithm 2. In case of asymptotic convergence to consensus, this method only allows an approximation of a common reference, without guaranteeing it exactly.

\section{Simulations}

To validate our algorithm we consider the search of a heat source in a 2-dimensional room $20 \times 12 \mathrm{~m}$ large; the walls are considered perfectly isolating, and we suppose to have an open window with an external temperature of $T_{\mathrm{ext}}=5{ }^{\circ} \mathrm{C}$, and a circular heater of $T_{\mathrm{s}}=50{ }^{\circ} \mathrm{C}$ in the middle. Our scenario is described by the problem with mixed Dirichlet-Neumann boundary conditions

$$
\begin{aligned}
f(z) & =T_{\mathrm{ext}} & & \text { on the window border } \\
f(z) & =T_{\mathrm{s}} & & \text { on the source border } \\
\nabla f(z) \cdot \hat{\boldsymbol{n}} & =0 & & \text { on the walls } \\
\nabla^{2} f(z) & =0 & & \text { inside the room, }
\end{aligned}
$$

with $\hat{\boldsymbol{n}}$ the outward unit normal to the wall.

To illustrate our algorithm, we consider a set of $n$ sensors with dynamics (1) and with the control law described in (6). The reference direction approximating the gradient is 


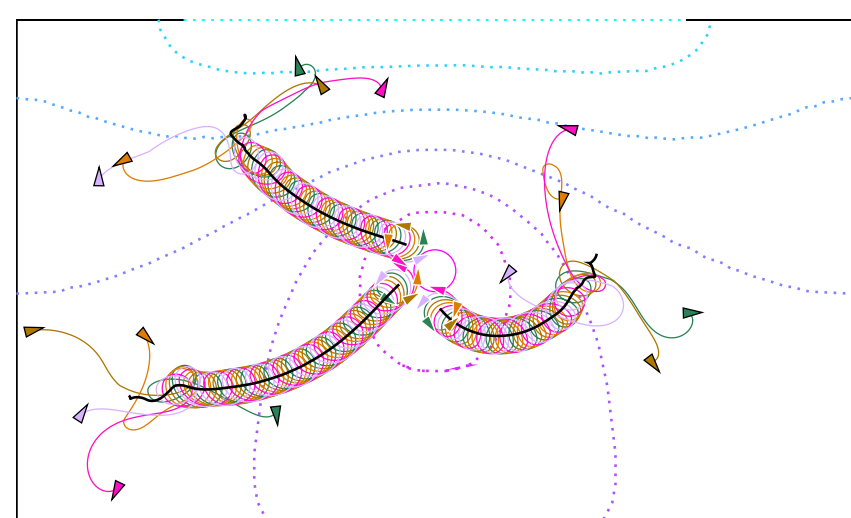

Fig. 5: Three simulations with five sensors in random initial positions.

computed with Algorithm 1, and its time derivative $\dot{\theta}_{\text {ref }}$ is obtained with discrete backward approximation.

Figure 5 shows the result of three simulations with $n=5$ sensors, starting from random initial positions and with random initial orientations, with velocities of $v_{\mathrm{r}}=0.5$ and $v_{\mathrm{c}}=0.1 \mathrm{~m} / \mathrm{s}$; the values for the control gain $k$ is chosen such to have a formation radius of $r=0.5 \mathrm{~m}$, i.e., $k=\frac{n v_{\mathrm{r}}}{\pi r} \approx$ 1.5915. We can see the trajectory of the sensors as well as the one of their centroid (in black); the black-bordered triangles represent the initial conditions of each sensor, while the whitebordered ones show their position and orientation when the formation reached the heater (the circle in the middle). The sensors reach a circular configuration soon, and then keep moving towards the source following the gradient of the field, approximately. Even if the theoretical convergence is local (for initial conditions near circle formation), the algorithm demonstrated to be effective also for randomly generated initial conditions. Moreover, despite the theoretical request to have the same common reference for each sensor in order to guarantee convergence, in practice Algorithm 1, which does not fulfill this assumption, gives good results.

Figures 6 and 7 show simulations similar to the ones in Figure 5, but illustrating the effect of noise and delay, respectively. In Figure 6, both temperature and bearing angle measurements are corrupted by white Gaussian noise of standard deviation $\sigma=0.5$. In Figure 7 the updates of the reference angle $\theta_{\text {ref }}$ happen with a delay of $1 \mathrm{~s}$, so as to show that the behaviour is not very degraded even in a case where distributed computation of $\theta_{\text {ref }}$ is heavily affected by delays in transmissions and computations.

Our algorithm can be applied with a different number of sensors, the minimum number being three, because two measurements are not enough to gather information about a 2dimensional gradient. In our simulations (not reported for lack of space), we have seen that three sensors are enough to have a nice behaviour, qualitatively similar to the one illustrated here with five sensors. A larger number of sensors ensures a better gradient approximation (at least when all sensors are in circular formation), but on the other hand the delay in the distributed computation of $\theta_{\text {ref }}$ grows linearly with $n$, due to the round of communications and updates in Algorithm 1. In

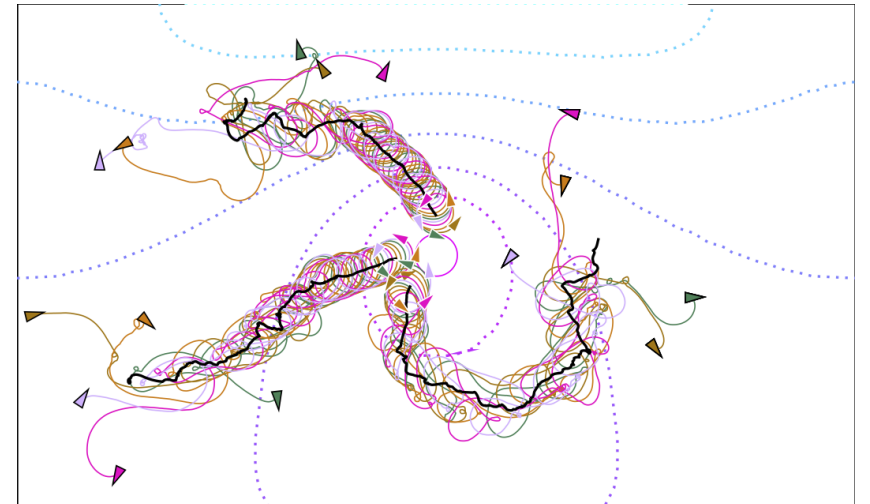

Fig. 6: Three simulations with five sensors in random initial positions, with noisy measurements.

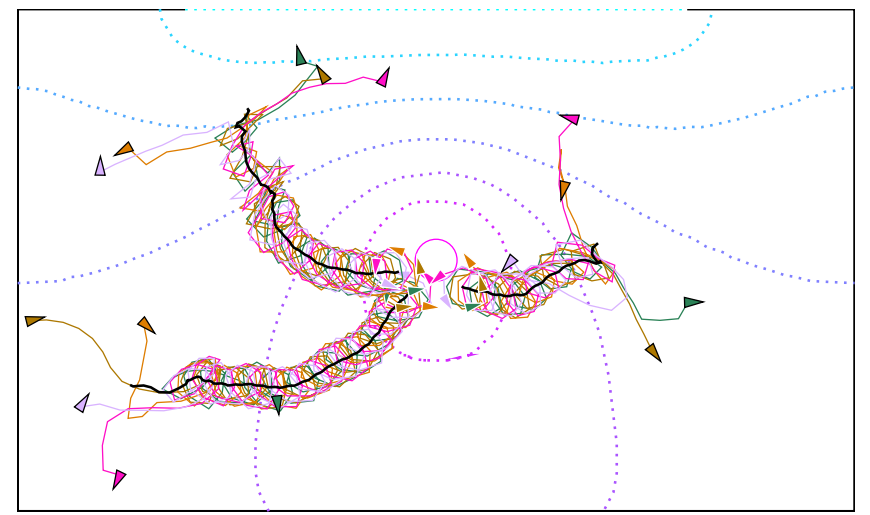

Fig. 7: Three simulations with five sensors in random initial positions, with delay in the computation of $\theta_{\text {ref }}$.

Figure 8, we show the trajectories obtained with different values of $n$, taking into account the corresponding delay. Sensors are initially placed in equispaced formation around a same circle, and we plot only the trajectory of the formation centroid. The parameters used in these simulations are $v_{\mathrm{r}}=0.5$ and $v_{\mathrm{c}}=0.1 \mathrm{~m} / \mathrm{s}$; the gain is $k=\frac{n v_{\mathrm{r}}}{\pi r}$ whith radius $r=1 \mathrm{~m}$. Due to the use of Algorithm 1, $\theta_{\text {ref }}$ is computed with a delay which grows linearly with $n$. However, the delay duration strongly depends on the software and hardware used for computation and communication. In Figure 8, we consider an example of application using MATLAB $^{\odot}$ on a general-purpose computer with a $2 \mathrm{GHz}$ clock, and an Arduino board. In this case, temperature acquisition and wireless IrDA transmission take negligible time, while computations (which need to be done sequentially: node $i$ waits for the result of the computation at node $i-1$ ), results in a total delay of approximately $5 n \mathrm{~ms}$. Such a delay does not have a major impact on the trajectories, and with growing $n$ the trajectory of the centroid becomes a better ${ }^{2}$ approximation of the ideal gradient ascent, which is plotted in black line for comparison. We have simulated a case

\footnotetext{
${ }^{2}$ In our simulations with same parameters as in Figure 8, we have always obtained that larger $n$ brings trajectories nearer to the ideal gradient ascent curve. In many cases, as in Figure 8, this also gives better trajectories, both in terms of time and distance necessary to reach the source. However, this is not true in general, in some cases a deviation from gradient ascent due to lower $n$ can also lead to a shorter trajectory.
} 


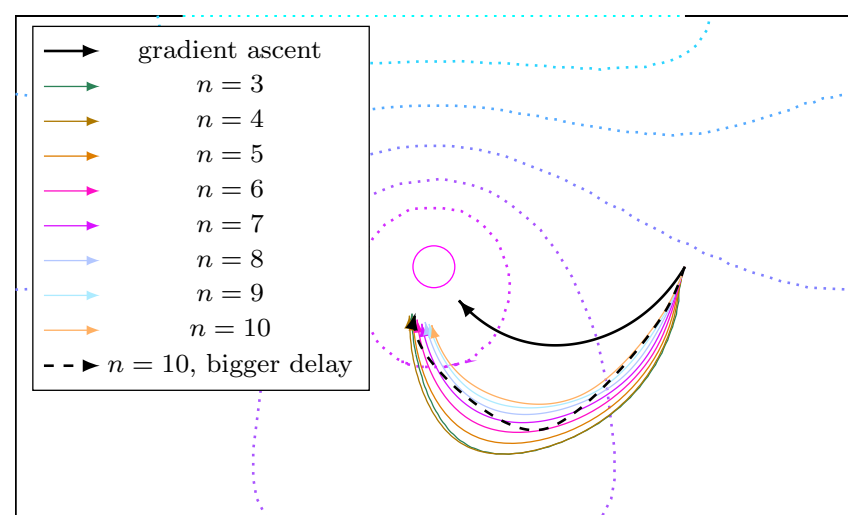

Fig. 8: Trajectories of the formation centroid, from initial equilibrium formation around a same circle, for different values of $n$. The computation of $\theta_{r}$ is affected by delay growing linearly with $n$, as $5 n \mathrm{~ms}$. Ideal gradient ascent is shown for comparison. Dashed line has $n=10$ and higher delay $25 n=250 \mathrm{~ms}$.

where delay grows more dramatically, as $25 n \mathrm{~ms}$ instead of $5 n \mathrm{~ms}$. For $n \leq 9$ this delay results in trajectories similar to the ones with smaller delay depicted in Figure 8, while for $n=10$ the centroid trajectory is visibly degraded, as shown by the dashed line added to the figure. This suggests to use a small number of sensors in applications with high delays. If a large number of sensors is necessary, e.g., because a large formation radius is required and communication range is small, it is still possible to mitigate the effect of delays, by suitably tuning the speed parameters $v_{\mathrm{c}}$ and $v_{\mathrm{r}}$, although at the price of a slower motion towards the source.

\section{CONCLUSIONS}

We considered the problem of localising a source by a set of communicating mobile sensors providing pointwise concentration measures and able to measure the relative bearing angle with respect to their neighbour. We have focused our attention on steady-state homogeneous isotropic diffusion processes, and developed a control law which allows the sensors to compute, in a distributed way, an estimation of the gradient argument of the function describing the diffusion of the measured quantity; such a control law did not use any explicit expression for the solution of the PDE, nor any global position information. The convergence of the source-seeking algorithm has been theoretically proven and, as an illustrative example, we have considered the search of a heat source in a 2-dimensional room.

\section{ACKNOWLEDGMENTS}

The authors would like to thank Antoine Rousseau for the FreeFem++ code used for the finite elements simulation.

\section{REFERENCES}

[1] L. Moreau, R. Bachmayer, and N. E. Leonard, "Coordinated gradient descent: a case study of Lagrangian dynamics with projected gradient information," in Proceedings of the 2nd IFAC Workshop on Lagrangian and Hamiltonian Methods in Nonlinear Control, Sevilla (Spain), April 2003, pp. 57-62.
[2] P. Ögren, E. Fiorelli, and N. E. Leonard, "Cooperative control of mobile sensor networks: adaptive gradient climbing in a distributed environment," IEEE Trans. Automatic Control, vol. 49, no. 8, pp. 1292$-1302,2004$.

[3] M. Rabbat and R. Nowak, "Distributed optimization in sensor networks," in Proceedings of the 3rd International Symposium on Information Processing on Sensor Networks, Berkeley (USA), 2004, pp. 20-27.

[4] B. J. Moore and C. Canudas-de-Wit, "Source-seeking via collaborative measurements by a circular formation of agents," in Proceedings of the 2010 IEEE American Control Conference, Baltimore (USA), 2010, pp. 6417-6422.

[5] L. Briñón Arranz, A. Seuret, and C. Canudas-de-Wit, "Collaborative estimation of gradient direction by a formation of AUVs under communication constraints," in Proceedings of the 50th IEEE Conference on Decision and Control and European Control Conference, Orlando (USA), December 2011, pp. 5583-5588.

[6] — " "Cooperative control design for time-varying formations of multiagent systems," IEEE Trans. Automatic Control, vol. 59, no. 8, pp. 22832288, 2014.

[7] R. Fabbiano, C. Canudas-de-Wit, and F. Garin, "Source localization by gradient estimation based on Poisson integral," Automatica, vol. 50, no. 6, pp. 1715-1724, June 2014.

[8] L. Briñón Arranz, L. Schenato, and A. Seuret, "Distributed sourceseeking via a circular formation of agents under communication constraints," IEEE Trans. Control of Network Systems, vol. 3, no. 2, pp. 104$115,2016$.

[9] N. Atanasov, J. Le Ny, and G. Pappas, "Distributed algorithms for stochastic source seeking with mobile robot networks," ASME Journal of Dynamic Systems, Measurement, and Control, vol. 137, 2015.

[10] W. Wu and F. Zhang, "Robust cooperative exploration with a switching strategy," IEEE Trans. Robotics, vol. 28, no. 4, pp. 828-839, 2012.

[11] E. Biyik and M. Arcak, "Gradient climbing in formation via extremum seeking and passivity-based coordination rules," Asian Journal of Control, vol. 10, no. 2, pp. 201-211, 2008.

[12] S. Z. Khong, Y. Tan, C. Manzie, and D. Nesic, "Multi-agent source seeking via discrete-time extremum seeking control," Automatica, vol. 50, no. 9, pp. 2312-2320, 2014.

[13] D. Scaramuzza, A. Martinelli, and R. Siegwart, "Precise bearing angle measurement based on omnidirectional conic sensor and defocusing," in European Conference on Mobile Robots (ECMR 2005), 2005.

[14] R. Fabbiano, C. Canudas-de-Wit, and F. Garin, "Distributed source localisation with no position information," in Proceedings of the 13th EUCA European Control Conference (ECC), Strasbourg (France), June 2014, pp. 569-574.

[15] N. Moshtagh, N. Michael, A. Jadbabaie, and K. Daniilidis, "Bearingonly control laws for balanced circular formations of ground robots," Robotics: Science and Systems IV, pp. 119-126, June 2008.

[16] J. A. Marshall, M. E. Broucke, and B. A. Francis, "Formations of vehicles in cyclic pursuit," IEEE Transactions on Automatic Control, vol. 49, no. 11, pp. 1963-1974, 2004.

[17] R. Fabbiano, "Collaborative source-seeking control," Ph.D. dissertation, Université Grenoble Alpes, May 2015. [Online]. Available: https://tel.archives-ouvertes.fr/tel-01217117

[18] F. Garin and L. Schenato, "A Survey on Distributed Estimation and Control Applications Using Linear Consensus Algorithms," in Networked Control Systems, A. Bemporad, M. Heemels, and M. Johansson, Eds. Springer, 2011, vol. 406, pp. 75-107.

[19] J. Hendrickx, G. Shi, and K. Johansson, "Finite-time consensus using stochastic matrices with positive diagonals," IEEE Trans. Automatic Control, vol. 60, no. 4, pp. 1070-1073, 2015.

[20] A. Dimakis, S. Kar, J. Moura, M. Rabbat, and A. Scaglione, "Gossip algorithms for distributed signal processing," Proceedings of the IEEE, vol. 98 , no. 11 , pp. 1847-1864, 2010. 